STRUCTURAL SCIENCE CRYSTAL ENGINEERING MATERIALS

ISSN 2052-5206

Received 27 November 2019

Accepted 31 March 2020

Edited by R. Černý, University of Geneva, Switzerland

Keywords: organic semiconductors; anthracene derivative compounds; single-crystal diffraction; synchrotron data; crystal structure; polymorphism; optical properties; luminescent compounds; laser confocal microscopy; fluorescence lifetime imaging microscopy.

CCDC references: $1962253 ; 1962254$

Supporting information: this article has supporting information at journals.iucr.org/b

\section{Synthesis, crystal structure, polymorphism and microscopic luminescence properties of anthracene derivative compounds}

\author{
Anna Moliterni, ${ }^{a *}$ Davide Altamura, ${ }^{a}$ Rocco Lassandro, ${ }^{a}$ Vincent Olieric, ${ }^{b}$ \\ Gianmarco Ferri, ${ }^{\text {c }}$ Francesco Cardarelli, ${ }^{c}$ Andrea Camposeo, ${ }^{\text {d }}$ Dario Pisignano, d,e \\ John E. Anthony ${ }^{f}$ and Cinzia Giannini ${ }^{a *}$
}

\begin{abstract}
${ }^{a}$ Istituto di Cristallografia, CNR, Via Amendola, 122/O, Bari, 70126, Italy, ${ }^{\mathbf{b}}$ Paul Scherrer Institute, Forschungstrasse 111, Villigen-PSI, 5232, Switzerland, ' $N E S T$, Scuola Normale Superiore, Piazza San Silvestro 12, Pisa, I-56127, Italy, dNEST, Istituto Nanoscienze, CNR, Piazza San Silvestro 12, Pisa, I-56127, Italy, 'Dipartimento di Fisica 'E. Fermi', University of Pisa, Pisa, I-56127, Italy, and 'Center for Applied Energy Research, University of Kentucky, Research Park Drive, Lexington, KY 2582, USA. *Correspondence e-mail: annagrazia.moliterni@ic.cnr.it, cinzia.giannini@ic.cnr.it
\end{abstract}

Anthracene derivative compounds are currently investigated because of their unique physical properties (e.g. bright luminescence and emission tunability), which make them ideal candidates for advanced optoelectronic devices. Intermolecular interactions are the basis of the tunability of the optical and electronic properties of these compounds, whose prediction and exploitation benefit from knowledge of the crystal structure and the packing architecture. Polymorphism can occur due to the weak intermolecular interactions, requiring detailed structural analysis to clarify the origin of observed material property modifications. Here, two silylethyne-substituted anthracene compounds are characterized by single-crystal synchrotron X-ray diffraction, identifying a new polymorph in the process. Additionally, laser confocal microscopy and fluorescence lifetime imaging microscopy confirm the results obtained by the X-ray diffraction characterization, i.e. shifting the substituents towards the external benzene rings of the anthracene unit favours $\pi-\pi$ interactions, impacting on both the morphology and the microscopic optical properties of the crystals. The compounds with more isolated anthracene units feature shorter lifetime and emission spectra, more similar to those of isolated molecules. The crystallographic study, supported by the optical investigation, sheds light on the influence of non-covalent interactions on the crystal packing and luminescence properties of anthracene derivatives, providing a further step towards their efficient use as building blocks in active components of light sources and photonic networks.

\section{Introduction}

Organic semiconductors have been a subject of growing interest during the last few decades due to their exploitation as active layers of a new generation of optoelectronic devices, such as organic light-emitting diodes (OLEDs; Yersin, 2008; Liu et al., 2013), organic solar cellar (OSCs; Palilis, et al., 2008; Dou et al., 2013; Ostroverkhova, 2016) and organic field-effect transistors (OFETs; Ito et al., 2003; Allard et al., 2008; Wang et al., 2012; Mei et al., 2013). Understanding their optoelectronic properties and the correlations with mechanical and morphological properties opened the door to the development of mechanically flexible, easily produced and cheap components for photonics, electronics, and energy conversion (Griffith et al., 2010). 
Among the organic semiconductors, acenes are aromatic hydrocarbons consisting of linearly fused benzene rings; the smallest compound of the acene family is anthracene, which can be easily obtained from anthracene oil, i.e. the coal-tar fraction that distils at a temperature above $270^{\circ} \mathrm{C}$. These compounds are especially interesting in their crystalline forms, which allow intriguing effects to be observed, such as polariton lasing (Kéna-Cohen et al., 2010). Intermolecular interactions play a major role in organic crystalline materials, not only determining molecular packing and, in turn, the optical and electrical properties of the solid-state material, but also its macroscopic properties, such as crystal habit, directly affecting light polarization, confinement and guiding (Camposeo et al., 2019). For example, needle-shaped crystals can exhibit optical waveguide properties with low propagation losses (Camposeo et al., 2019). $\pi-\pi$ interactions favour an efficient channel for charge mobility (Anthony et al., 2002; Chen et al., 2006; Yao et al., 2018) and are, therefore, exploited in material design (da Silva Filho et al., 2005). It is also known that non-covalent self-assembly of acenes can be controlled by the insertion of suitable molecular substituents, by which crystal properties can be effectively tailored (Anthony, 2006, 2008). Therefore, engineering of the active material relies on the in-depth knowledge of crystal structure and non-covalent interactions. Here, we provide such a detailed characterization through single-crystal synchrotron X-ray diffraction for two silylethyne-substituted anthracene compounds, i.e. 1,2,3,4-tetrafluoro-5,8-bis(trimethylsilylethynyl)anthracene and 9,10bis(triisopropylsilylethynyl)anthracene (F4 TMS ANT and TIPS ANT, respectively), previously studied by Camposeo et al. (2019) and Anthony \& Parkin (2016), respectively. The structural characterization of F4 TMS ANT carried out by Camposeo et al. (2019) is confirmed and improved in terms of structure model accuracy, and a detailed description of the intermolecular interactions is provided as well. In addition, a new polymorph of TIPS ANT is identified (named TIPS ANT $p$ in this work), revealing that different molecular packing can result from the same synthesis procedure (Landis et al., 2005). The occurrence of polymorphism in the case of TIPS anthracene compounds has also been observed recently by Bhattacharyya \& Datta (2017). The position of the molecular substituents towards the external benzene rings is shown to influence the crystal morphology (i.e. needle and plate shape in the case of F4 TMS ANT and TIPS ANT $p$, respectively) and the strengths of the $\pi-\pi$ interactions.

Laser confocal microscopy and fluorescence lifetime imaging microscopy show for the two compounds significant differences in the luminescence properties, along with the uniform emission throughout for both compounds. The lifetime measurements performed here show decay times of the photoluminescence (PL) an order of magnitude longer for $\mathrm{F} 4$ TMS ANT with respect to TIPS ANT $p$. The dissimiliar PL features of the two compounds are in agreement with their dissimilar molecular arrangement suggested by the crystallographic study.

The accurate structural description here provided is a further step in view of tailoring crystal morphology and optical properties to achieve the sought compromise between molecular stabilization and optimal performance of the organic semiconductor (Gu et al., 2012) and/or its coupling to an optical network (Camposeo et al., 2019).

\section{Experimental}

\subsection{Synthesis and crystallization}

Synthesis of F4 TMS ANT has been described in (Camposeo et al., 2019); the synthesis method for obtaining TIPS ANT $p$ has been reported by Landis and co-workers (Landis et al., 2005).

\subsection{X-ray diffraction}

Single-crystal X-ray diffraction data were collected at the beamline PXIII (X06DA-PXIII, http://www.psi.ch/sls/pxiii/) at the Swiss Light Source (SLS), Villigen, Switzerland, using a Parallel Robotics Inspired (PRIGo) multi-axis goniometer (Waltersperger et al., 2015) and a PILATUS 2M-F detector. Data collections were performed at room temperature $(T=$ 296 K) on selected crystals of F4 TMS ANT and TIPS ANTp, mounted on litholoops (Molecular Dimensions). For each crystal, complete data were obtained by merging two $360^{\circ} \omega$ scans at $\chi=0^{\circ}$ and $\chi=30^{\circ}$ of PRIGo. In shutterless mode, a $360^{\circ}$ data set was collected in $3 \mathrm{~min}$ (beam energy of $17 \mathrm{keV}$, $\lambda=0.72932 \AA$, focus size $90 \mu \mathrm{m} \times 50 \mu \mathrm{m}, 0.25 \mathrm{~s}$ of exposure time per frame, $0.5^{\circ}$ scan angle).

Main data collection details are given in Table 1. Partial data sets were individually processed by $X D S$ (Kabsch, 2010), a software organized in eight subroutines able to carry out the main data reduction steps; the corresponding XDS_ASCII.HKL reflection files were scaled and merged by the XSCALE subroutine (Kabsch, 2010). Structure solution was carried out by direct methods using SIR2019 (Burla et al., 2015) and refined by SHELXL2014/7 (Sheldrick, 2015). All non-hydrogen atoms were refined anisotropically. The carbonbound $\mathrm{H}$ atoms were placed on geometrically calculated positions and refined using a riding-model approximation.

\subsection{Laser confocal microscopy and fluorescence lifetime imaging microscopy}

The microscale optical properties of F4 TMS ANT and TIPS ANT $p$ were investigated by laser confocal microscopy (LCM). To this aim, spatially resolved emission spectra were measured by an inverted microscope equipped with a laser confocal scanning head (FV1000, Olympus), by exciting samples with a continuous wave laser emitting at $405 \mathrm{~nm}$ through either a $10 \times$ objective (Olympus UPLSAPO) with 0.4 numerical aperture (NA) or a $60 \times$ objective (Olympus PLAPON) with $\mathrm{NA}=1.42$. The excitation power was in the range $10-50 \mu \mathrm{W}$. The laser was focused to a diffraction-limited spot on to the sample. The photoluminescence was collected by the same objective and measured by a photomultiplier (Olympus). The sampling speeds during the measurements of the fluorescence micrographs were in the interval $15-80 \mu \mathrm{s} \mu \mathrm{m}^{-1}$. Typically, higher excitation power and sampling speed were used for F4 
Table 1

Experimental and refinement details for F4 TMS ANT and TIPS ANT $p$.

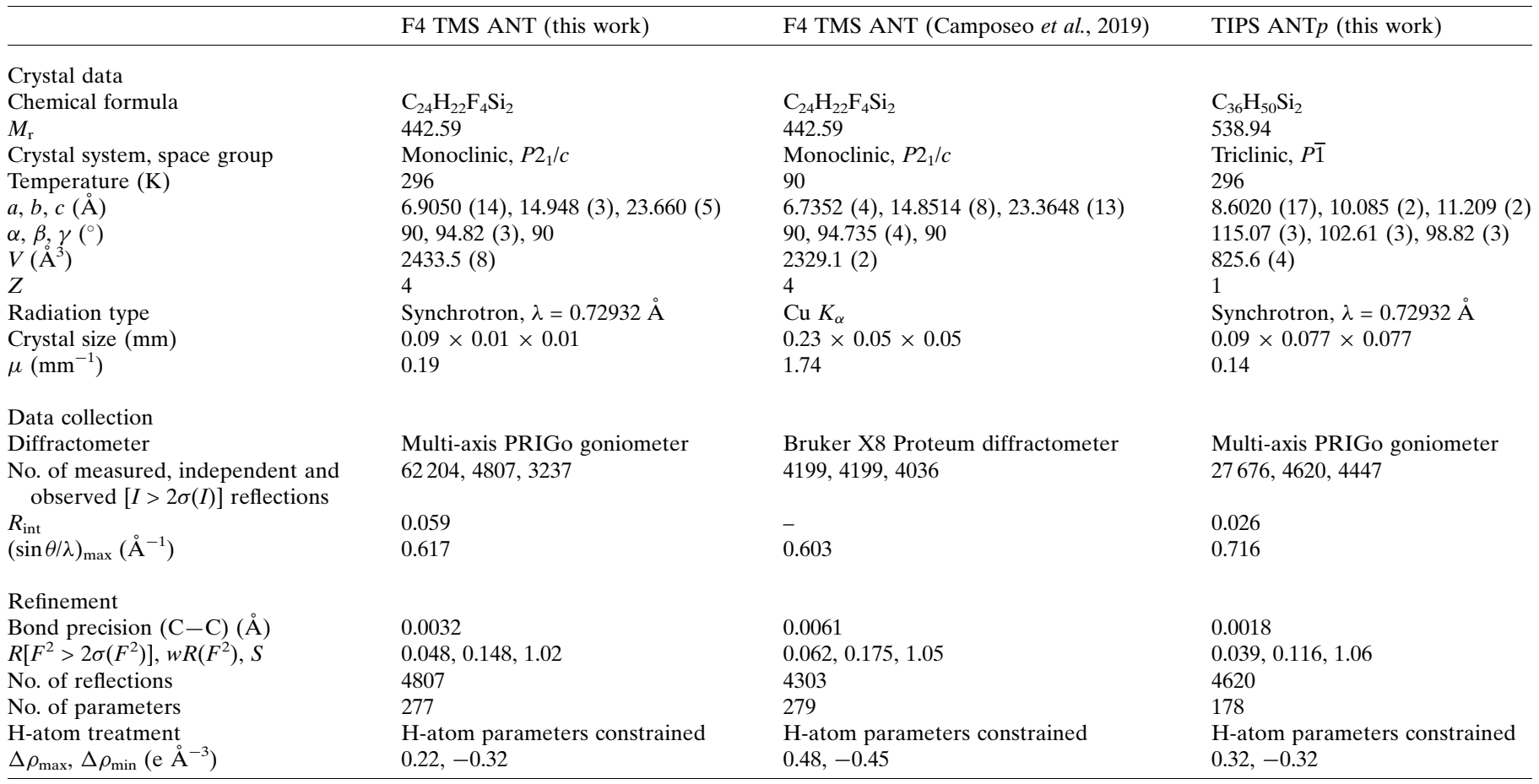

Computer programs: XDS (Kabsch, 2010), SIR2019 (Burla et al., 2015), SHELXL2014/7 (Sheldrick, 2015), WinGX (Farrugia, 2012), publCIF (Westrip, 2010), Mercury (Macrae et al., 2008) and CrystalExplorer17 (Turner et al., 2017).

TMS ANT samples. In order to measure the spatially resolved PL spectra, confocal micrographs were collected at various emission wavelengths with a spectral bandwidth of $2 \mathrm{~nm}$. A polarization analyser positioned on the optical path of the emission was used for measuring polarized PL spectra. To this aim, samples were excited by a linearly polarized laser, with polarization direction parallel to the long axis of the TIPS ANT $p$ platelets. The PL lifetime for the crystalline samples was investigated by confocal fluorescence lifetime imaging microscopy (FLIM). This analysis was carried out on an inverted microscope with a confocal head (TCS SP5, Leica Microsystem $)$ and either a $40 \times(\mathrm{NA}=1.25)$ or a $100 \times$ objective (Fluotar, NA =1.3). Samples were optically excited by a $405 \mathrm{~nm}$ pulsed diode laser (Picoquant, Sepia Multichannel picosecond diode laser, maximum average power $30 \mu \mathrm{W}$ ) operating at $40 \mathrm{MHz}$, whereas the fluorescence intensity was measured by a photomultiplier tube interfaced with a time-correlated single photon counting setup (PicoHarp 300, PicoQuant, Berlin). The detection rate was kept in the interval $10^{5}-10^{6}$ counts per second, while lifetime signals were time-integrated until reaching an average value in the order of $10^{2}$ counts in each area of the scanned micrographs. Spectral filters with variable bandwidths were exploited in order to measure the PL lifetime in different spectral intervals. The measured temporal decay profiles of the PL were fitted to exponential functions convoluted with the instrumental response function, that is assumed to be Gaussian with fullwidth at half maximum of $280 \mathrm{ps}$.
For confocal analysis the crystalline samples were placed on top of a glass coverslip (thickness $150 \mu \mathrm{m}$ ), while for macroscopic optical characterization the crystalline samples were placed on the surface of $1 \mathrm{~cm} \times 1 \mathrm{~cm}$ quartz substrates (thickness $1 \mathrm{~mm}$ ). Crystalline samples with low inhomogeneities as visible by bright and dark field optical microscopy were selected for the measurements. Absorption spectra were measured with a UV-visible spectrophotometer (Lambda 950, Perkin Elmer). The samples were mounted on a sample holder for solid state specimens. The sample holder was a metallic plate with a central clear part and two clamps which block two edges of the quartz substrate, while leaving the central part of the substrate free for optical access. The incident optical beam was properly masked in order to have a spot size matching the area of the crystalline sample.

\subsection{Structure solution and refinement}

Both F4 TMS ANT and TIPS ANTp were solved and refined from single-crystal synchrotron X-ray diffraction data. According to the literature, TIPS ANT crystallized in the centrosymmetric space group $P b c a$ and the related crystallographic data were deposited at the Cambridge Crystallographic Data Centre (CCDC), with deposition number CCDC 962668 (Anthony \& Parkin, 2016); in the case of TIPS ANT, data collection was carried out at a safe temperature of $T=250 \mathrm{~K}$ because the authors observed that a destructive phase transition occurred for crystals cooled to $240 \mathrm{~K}$. In the 
present work, in order to investigate the occurrence of phase transitions caused by cooling, for TIPS ANT $p$ two diffraction experiments were carried out, at room temperature and at $250 \mathrm{~K}$, respectively. The analysis of the corresponding sets of diffraction data revealed no changes in the crystal structure. Consequently, the results presented here concern only roomtemperature measurements.

The structure characterization presented in this work in the case of F4 TMS ANT confirmed the crystal structure results described by Camposeo et al. (2019), [whose corresponding CIF file was deposited at the Cambridge Crystallographic Data Centre (CCDC) with deposition number CCDC 1838578 and for the sake of completeness provided also as supplementary material (i.e. file 1838578.cif)] while for TIPS ANTp enabled to identify a new polymorph of TIPS ANT, that crystallized in the centrosymmetric space group $P \overline{1}$.

The crystal structure solution step was carried out by SIR2019 (Burla et al., 2015) that exploits the information on cell parameters, diffraction intensities and expected chemical formula to determine the space group and solve the structure by direct methods (Giacovazzo, 2014). Crystal structures were refined using full-matrix least-squares techniques by SHELXL2014/7 (Sheldrick, 2015). Non-hydrogen atoms were refined anisotropically while hydrogen atoms were geometrically positioned and constrained to ride on their parent $\mathrm{C}$ atoms with the following bond lengths constraints: $\mathrm{C}-\mathrm{H}=$ $0.96 \AA$ and $\mathrm{C}-\mathrm{H}=0.93 \AA$ for methyl and aromatic $\mathrm{H}$ atoms, respectively. The isotropic $U$ value constraint $U_{\text {iso }}(\mathrm{H})=k$ $U_{\text {eq }}(\mathrm{C})$ was set, with $k=1.5$ and 1.2 for methyl and aromatic $\mathrm{H}$ atoms, respectively; a rotating group model was applied for methyl groups.

Main crystal data and details on data collection and structure refinement are summarized in Table 1 that, in the case of

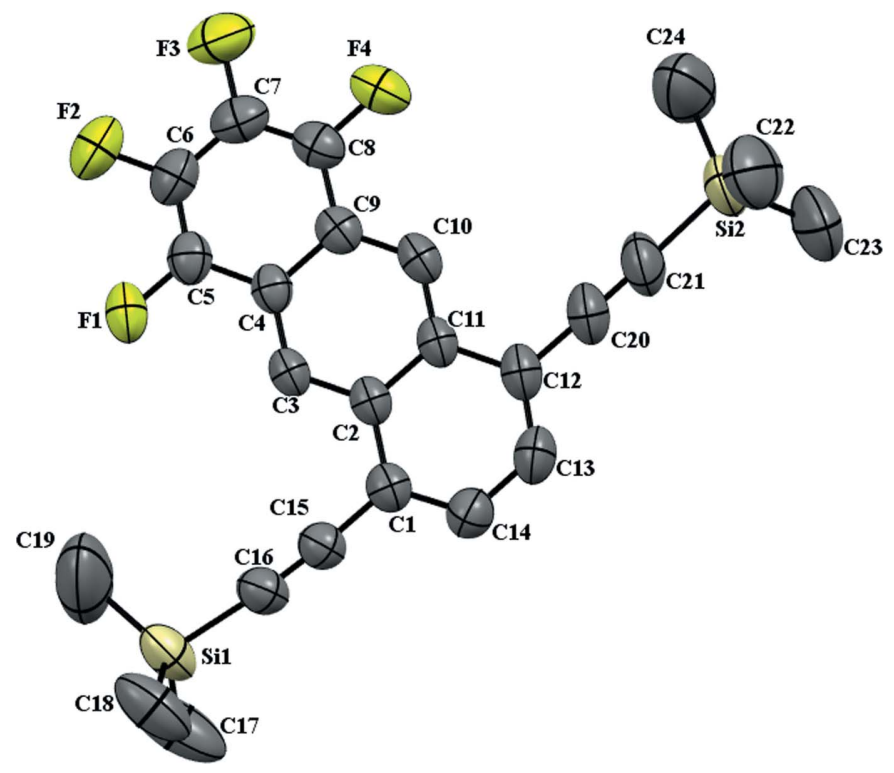

Figure 1

F4 TMS ANT: A view along $a$ of the asymmetric unit with the atomic labelling scheme. $\mathrm{H}$ atoms are omitted for clarity. Ellipsoids are drawn at the $50 \%$ probability level.
F4 TMS ANT, provides also the results obtained by Camposeo et al. (2019) (see the corresponding CIF file) for the sake of comparison.

\section{Results and discussion}

F4 TMS ANT crystallized in the centrosymmetric space group $P 2_{1} / c$, with one molecule in the asymmetric unit (see Fig. 1), and TIPS ANT $p$ crystallized in the centrosymmetric space group $P \overline{1}$, with half a molecule in the asymmetric unit (see Fig. 2). In the case of F4 TMS ANT the space group determination was automatically carried out by SIR2019 by considering the Laue group compatible with the geometry of the unit cell and assigning a probability value to each related extinction symbol, taking into account a statistical analysis carried out on the experimental intensities; at the end of this step the most plausible space group was graphically selected. For both compounds, the structure solution process was automatically performed by SIR2019. Crystal structure refinement was carried out by SHELXL2014/7 by applying full-matrix least-squares techniques. Non-hydrogen atoms were refined anisotropically while a riding-model approximation was applied in the case of hydrogen atoms: $\mathrm{H}$ atoms were geometrically positioned at the bond distances $\mathrm{C}-\mathrm{H}=$ $0.96 \AA$ and $\mathrm{C}-\mathrm{H}=0.93 \AA$ for methyl and aromatic $\mathrm{H}$ atoms, respectively and allowed to ride on their respective parent $\mathrm{C}$ atoms. In the case of methyl group, a rotating group model was assumed and the torsion angle defining its orientation about the $\mathrm{Si}-\mathrm{C}$ bond [in the case of F4 TMS ANT] or the $\mathrm{C}-\mathrm{C}$ bond [in the case of TIPS ANTp], was refined. The isotropic $U$ value satisfied the following constraints: $U_{\text {iso }}(\mathrm{H})=k U_{\text {eq }}(\mathrm{C})$, with $k=1.5$ and 1.2 for methyl and aromatic $\mathrm{H}$ atoms, respectively.

Main crystallographic data are given in Table 1; additional tables, concerning refined fractional atomic coordinates and displacement parameters, bond distances and angles, and torsion angles, were provided in the supporting information.

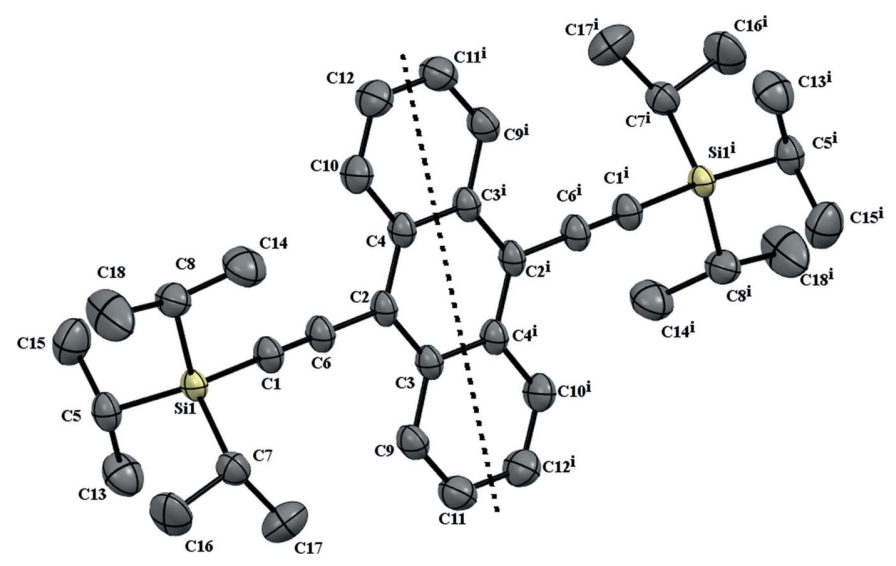

Figure 2

TIPS ANT $p$ : a view of the asymmetric unit (half of molecule, drawn on the left-hand side of the broken line), showing the atom-labelling scheme. $\mathrm{H}$ atoms are omitted for clarity. Ellipsoids are drawn at the $50 \%$ probability level. [Symmetry code: (i) $-x, 1-y,-z$ ]. 


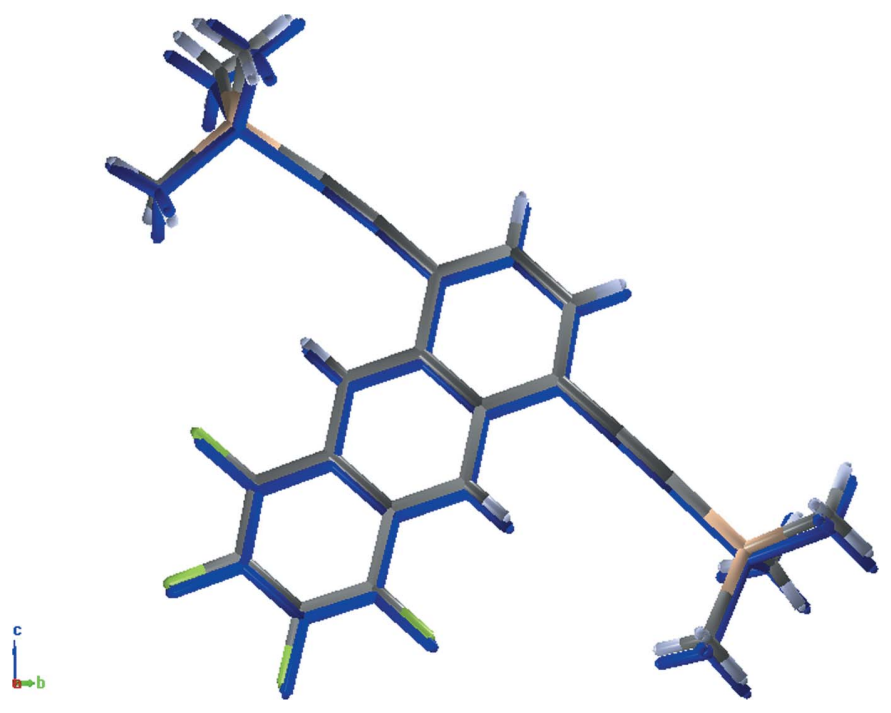

Figure 3

F4 TMS ANT: A view of the structural overlay of the refined crystal structures, in case of the present work and of the published structure by Camposeo et al. (2019) (this last one is shown in blue colour) having an r.m.s. deviation of $0.04 \AA$ (SIR2019; Burla et al., 2015).

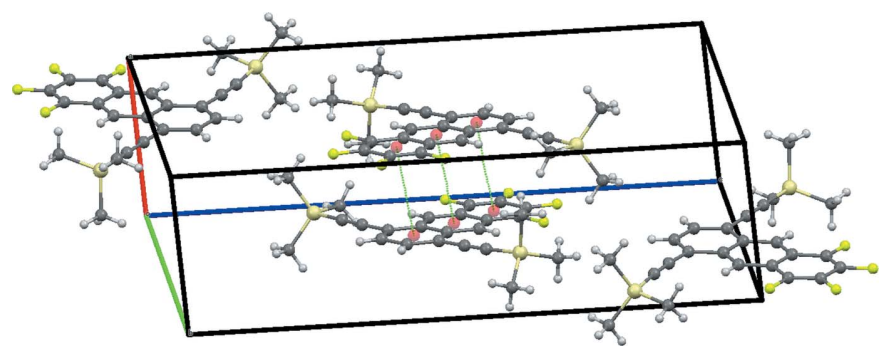

Figure 4

F4 TMS ANT: a view of crystal packing showing the parallel-offset $\pi-\pi$ interactions that are indicated by green broken lines between centroids of the aromatic rings (represented with red spheres).
For both compounds no presence of classical hydrogen bonds was detected; the crystal packing suggested that the main intermolecular interactions were weak and, in the case of F4 TMS ANT, consisted of $\pi-\pi$ interactions (Janiak, 2000; Meyer et al., 2003; Martinez \& Iverson, 2012) and $\mathrm{C}_{\text {aryl }}-$ F...H-C interactions (Meyer et al., 2003); for TIPS ANTp, they were aromatic interactions, weaker than those involved in F4 TMS ANT, and C $-\mathrm{H} \cdots \pi$ interactions (Meyer et al., 2003; Nishio, 2004; Nishio et al., 2012).

In the case of F4 TMS ANT, the refined crystal structure here described is similar to that obtained by Camposeo et al. (2019), as shown in Fig. 3, providing the overlay of the two structure solutions, having an r.m.s. deviation of $0.04 \AA$ (SIR2019; Burla et al., 2015). Our results, compared to the structure model by Camposeo et al. (2019), are characterized by a significant improvement in terms of $\mathrm{C}-\mathrm{C}$ bond precision and agreement factors; the bond precision, in the case of our results, is $0.0032 \AA$ instead of $0.0061 \AA$, while the $R\left[F^{2}>\right.$ $\left.2 \sigma\left(F^{2}\right)\right]$ and $w R\left(F^{2}\right)$ values were 0.048 and 0.148 instead of 0.062 and 0.175 , respectively (see Table 1 ).

For F4 TMS ANT (Fig. 1), the anthracene core consists of three planar-like six-membered rings. The mean distance from the least-squares plane calculated for 18 atoms, including the $14 \mathrm{C}$ atoms from the anthracene group and the four $\mathrm{F}$ atoms, was $0.0074 \AA$ and the largest deviation was at C14 (0.023 $\AA$ ) and at F4 (0.019 $\AA$ ). A slight bending was observed for the lateral chains involving the alkyne group: the distances from the least-squares plane for C15, C16 and Si1 were 0.024 (1), 0.047 (2) and 0.117 (2) A, respectively, while for C20, C21 and Si2 were 0.074 (2), 0.135 (3) and 0.280 (3) $\AA$, respectively. As usual for anthracenyl units, the peripheral aromatic rings were distorted from the hexagonal geometry with shortening of some bond distances (Kovalski et al., 2018): the bond distances C5-C6, C7-C8, C1-C14, C12-C13 were $1.341(3)$, 1.346 (3), 1.378 (3) and 1.364 (3) Å, respectively, while the rest

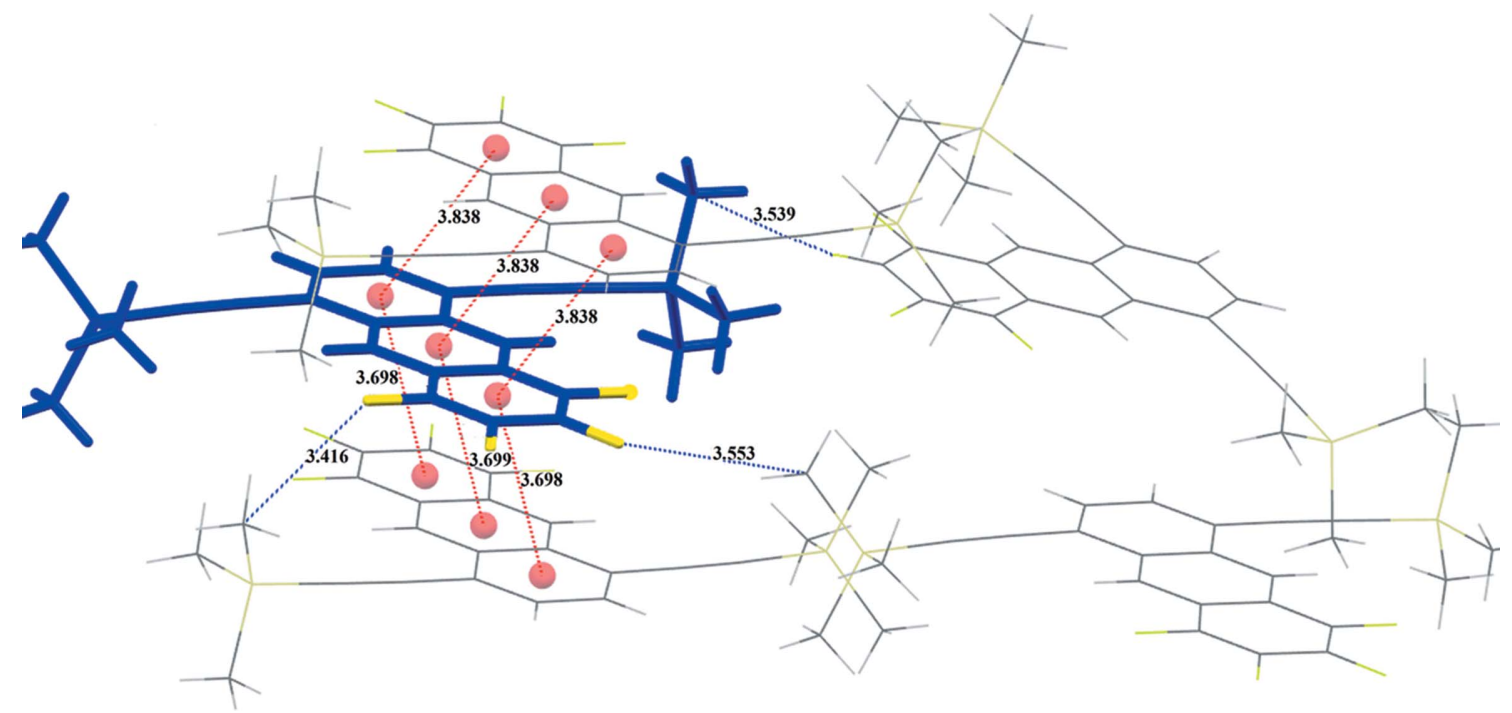

Figure 5

F4 TMS ANT: a view of the local environment of the molecule represented with sticks and its neighbouring entities (represented with wireframes); centroids are represented with red spheres. The $\pi-\pi$ interactions are indicated by broken red lines while $\mathrm{C}_{\text {aryl }}-\mathrm{F} \cdots \mathrm{H}-\mathrm{C}$ interactions by broken blue lines. Distances given in $\AA$. 
of bond distances were between 1.389 (3) and 1.445 (3) A. The mean value of the $\mathrm{C}-\mathrm{C}$ bonds was 1.4098 (30) $\AA$ (ring $\mathrm{C} 1-$ $\mathrm{C} 2-\mathrm{C} 11-\mathrm{C} 12-\mathrm{C} 13-\mathrm{C} 14), 1.4042$ (30) $\AA$ (ring $\mathrm{C} 2-\mathrm{C} 3-$ $\mathrm{C} 4-\mathrm{C} 9-\mathrm{C} 10-\mathrm{C} 11)$ and 1.3938 (30) $\AA$ (ring $\mathrm{C} 4-\mathrm{C} 5-\mathrm{C} 6-$ $\mathrm{C} 7-\mathrm{C} 8-\mathrm{C} 9)$. The crystal packing showed the presence of weak $\mathrm{C}_{\text {aryl }}-\mathrm{F} \cdots \mathrm{H}-\mathrm{C}$ interactions (Meyer et al., 2003) and parallel offset $\pi-\pi$ interactions (Janiak, 2000; Meyer et al., 2003; Martinez \& Iverson, 2012), as shown in Figs. 4 and 5. These kinds of $\pi-\pi$ arrangements are energetically more stable and favoured than the parallel face-centred stacked

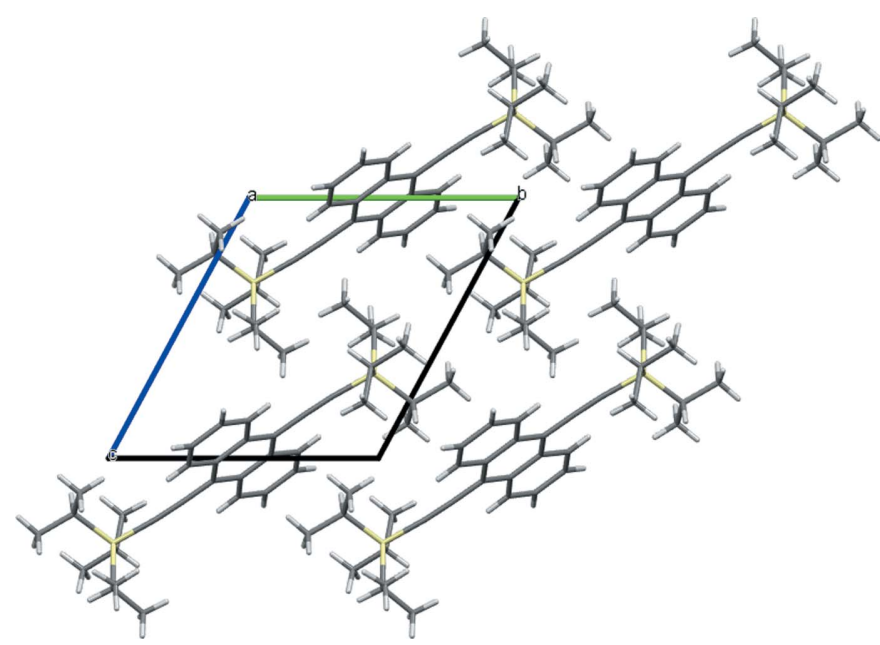

Figure 6

TIPS ANT $p$ : a view along $a$ of the crystal packing. ones (Martinez \& Iverson, 2012). The minimum distance between centroids of the aromatic rings was 3.698 (3) $\AA$ (see Fig. 5) and the minimum interplanar distance was 3.422 (3) $\AA$, in agreement with the typical values of the interplanar distances for $\pi-\pi$ interactions, belonging to the range 3.3$3.8 \AA$ (Janiak, 2000). The parallel offset $\pi-\pi$ interactions were responsible for stacking arrangements along $a$ (see Fig. 4). $\mathrm{C}_{\text {aryl }}-\mathrm{F} \cdots \mathrm{H}-\mathrm{C}$ interactions contributed to stabilize the crystal structure (Meyer et al., 2003) and the values of the distance F . . C belonged to the range observed for this kind of interaction [i.e. 3.30 (1)-3.60 (1) A; see Meyer et al. (2003)].

In the case of TIPS ANTp, the mean distance from the leastsquares plane, calculated for the $\mathrm{C}$ atoms of anthracene group, was 0.0041 (3) $\AA$ and the largest deviation was at C3

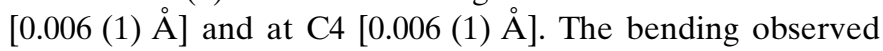
for the lateral chain involving the alkyne group was larger than in the case of F4 TMS ANT, probably due to the larger number of methyl groups: the distances from the least-squares plane for C6, C1 and Si1 were 0.048 (2), 0.136 (2) and 0.409 (2) $\AA$, respectively. Also for TIPS ANTp the peripheral aromatic rings were distorted from the hexagonal geometry: the bond distances $\mathrm{C} 10-\mathrm{C} 12$ and $\mathrm{C} 9-\mathrm{C} 11$ were 1.3580 (17) and $1.3561 \AA(16)$, respectively, while the rest of bond distances were between 1.4106 (14) and 1.4300 (12) A. The mean value of the $\mathrm{C}-\mathrm{C}$ bonds in the three aromatic rings shown in Fig. 2 is 1.4005 (15) $\AA$ (ring $\mathrm{C} 4-\mathrm{C} 10-\mathrm{C} 12-\mathrm{C} 11^{\mathrm{i}}-$ $\left.\mathrm{C} 9^{\mathrm{i}}-\mathrm{C} 3^{\mathrm{i}}\right), 1.4173(13) \AA$ ( 1 ring $\mathrm{C} 3-\mathrm{C} 2-\mathrm{C} 4-\mathrm{C} 3^{\mathrm{i}}-\mathrm{C} 2^{\mathrm{i}}-\mathrm{C} 4^{\mathrm{i}}$ ) and 1.4005 (15) $\AA$ (ring $\mathrm{C} 11-\mathrm{C} 9-\mathrm{C} 3-\mathrm{C} 4^{\mathrm{i}}-\mathrm{C} 10^{\mathrm{i}}-\mathrm{C} 12^{\mathrm{i}}$ ). $\mathrm{A}$ view along $a$ of the crystal packing is given in Fig. 6. In contrast

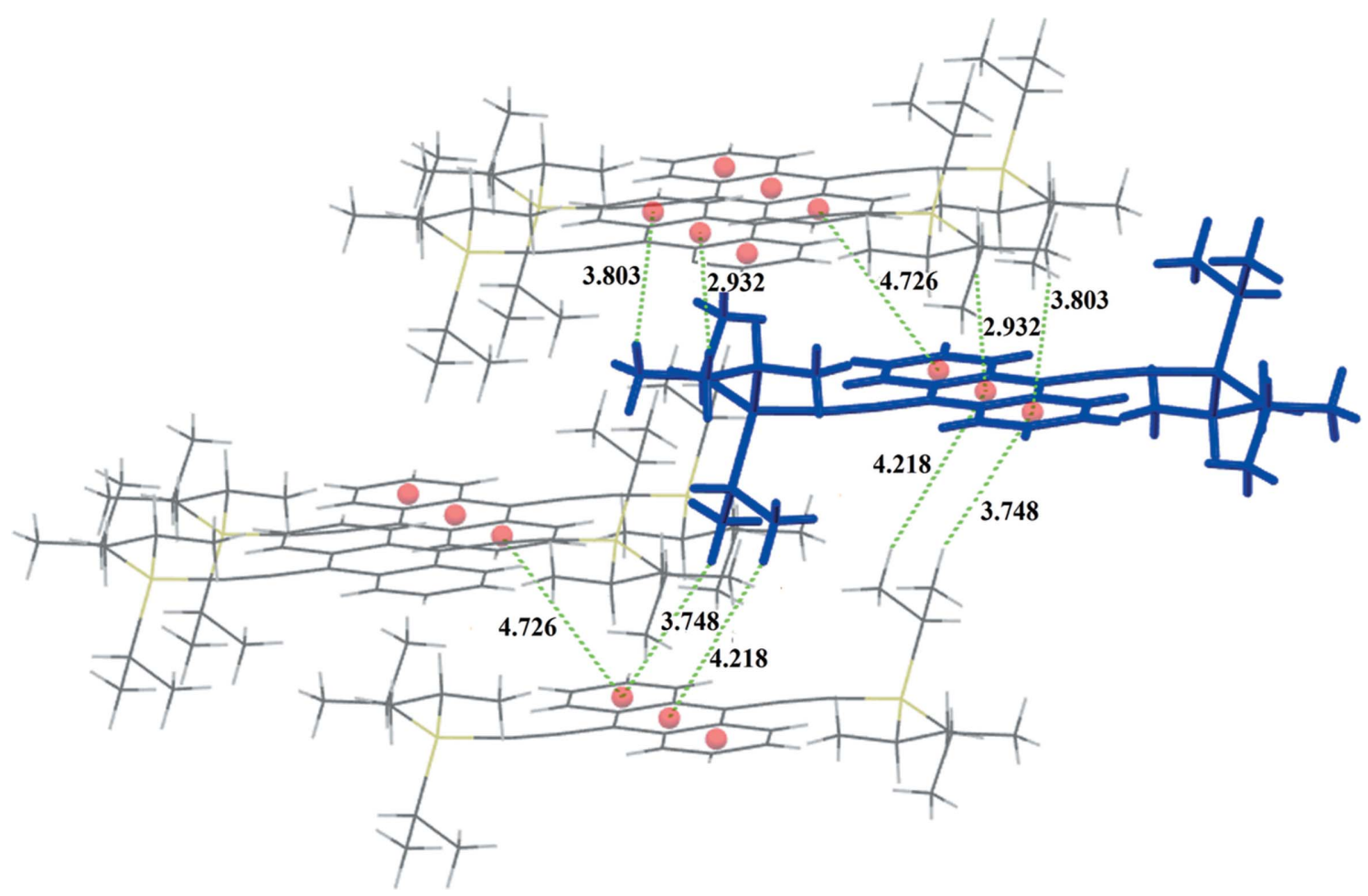

Figure 7

TIPS ANTp: a view of the local environment of the molecule represented with sticks and its neighbouring entities (represented with wireframes); centroids are represented with red spheres. The weak $\pi-\pi$ interactions (with a distance between centroids of $4.726 \AA$ ) are indicated by broken green lines as well as the $\mathrm{C}-\mathrm{H} \cdots \pi$ interactions. Distances given in $\AA$. 
to TIPS ANT (Anthony \& Parkin, 2016), the crystal packing in TIPS ANT $p$ (present work) did not reveal any edge-to-face interaction; the main intermolecular contacts were weak interactions between parallel aromatic rings and $\mathrm{C}-\mathrm{H} \cdots \pi$ interactions (Meyer et al., 2003; Nishio, 2004; Nishio et al., 2012). The last ones, as observed by Nishio (2004), are entropically favoured and contribute to stabilize the crystal structure. The parallel aromatic rings are characterized by a large offset [the shortest distance between centroids of parallel aromatic rings is $4.726(2) \AA$, see Fig. 7, and the minimum interplanar distance is 2.471 (2) $\AA$ ]; consequently, these interactions, in spite of the short interplanar distance, were weaker than the parallel offset $\pi-\pi$ interactions detected in F4 TMS ANT, and due to the large distance between centroids are not the typical $\pi-\pi$ interactions (Janiak, 2000). If compared with F4 TMS ANT, the weaker $\pi-\pi$ interactions in the case of TIPS ANT $p$ could lead to a reduction of the charge mobility, which should be confirmed by proper electrical characterization (which is beyond the goal of this paper). The values of $\mathrm{C}-\mathrm{H} \cdots \pi$ distances shown in Fig. 7 belonged to the distance range observed for this kind of interaction [i.e. 3.3 (1)-4.1 (1) ̊, see Meyer et al. (2003), Hattab et al. (2010)].

The main intermolecular interactions for F4 TMS ANT and TIPS ANT $p$ crystal structures can be represented via Hirshfeld surfaces, using the CrystalExplorer17 software (Turner $e t$ al., 2017). The Hirshfeld surface offers a useful tool for measuring the space occupied by a molecule in a crystal and summarizing information on all intermolecular interactions. In

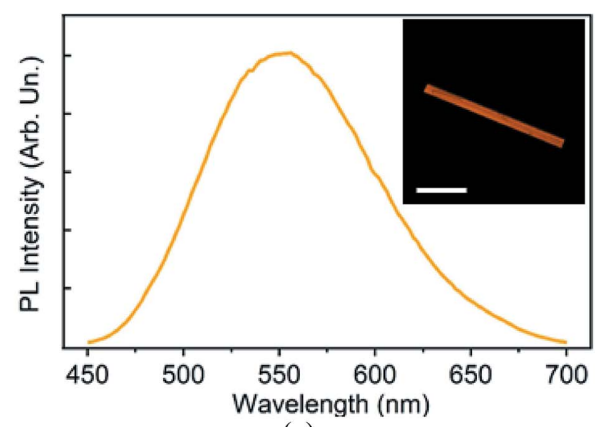

(a)

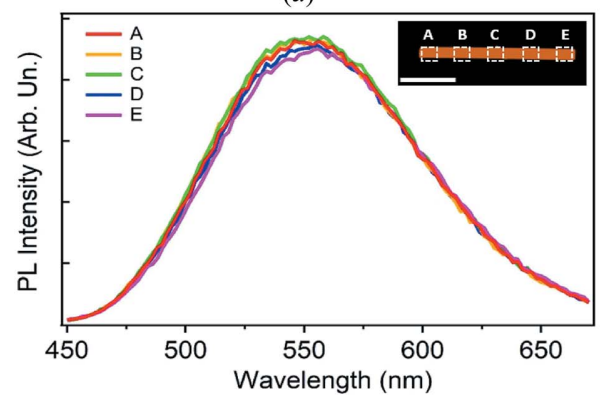

(c)

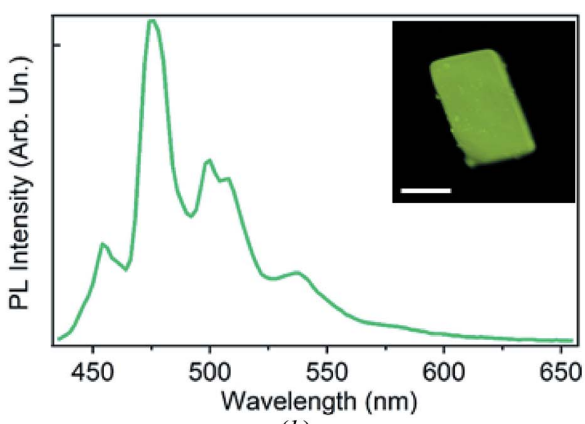

(b)

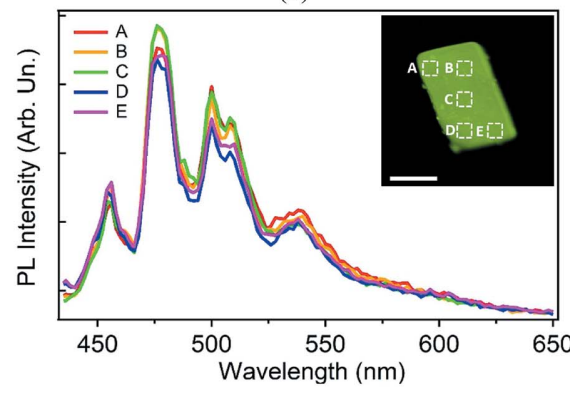

(d)
Figure 8

PL spectra of F4 TMS ANT (a) and TIPS ANT $p(b)$, measured by confocal microscopy. The corresponding micrographs of the PL intensity of the investigated samples are shown in the insets. Inset scale bars: $50 \mu \mathrm{m}$. Comparison of PL spectra of F4 TMS ANT (c) and TIPS ANT $p(d)$, measured by confocal microscopy in various regions of the crystalline samples. The corresponding insets show confocal PL micrographs of the investigated samples. Scale bars: $50 \mu \mathrm{m}$. The spectra (A-E) are measured in the areas marked with the dashed squares in the insets. The spectra shown in $(c)$ and $(d)$ and the PL micrographs were acquired using the objective with NA $=0.4$.
Figs. S1 and S2 the Hirshfeld surface mapped over $d_{\text {norm }}$ is shown for F4 TMS ANT and TIPS ANT $p$, respectively. The conventions for the surface colours are as follows: blue, white and red colours identify the interatomic contacts as longer, at van der Waals separations and short, respectively. In both figures the blue colour is predominant. No red region is observed for TIPS ANT $p$ whereas for F4 TMS ANT a very small and faint red region, indicated by red arrows in Fig. S1a and zoomed in Fig. S1b, corresponds to the $\mathrm{C}_{\text {aryl }}-\mathrm{F} \cdots \mathrm{H}-\mathrm{C}$ interactions.

The different arrangement and molecular interactions of the anthracene derivatives in the crystalline samples also affect the PL of the single crystals. Fig. 8 compares the microscopic PL emission spectra of individual single crystals of F4 TMS ANT and TIPS ANT $p$, as measured by laser confocal microscopy. TIPS ANT $p$ crystals feature brighter emission, typically requiring lower laser power for optical excitation (by about a factor 5) and sampling speeds (by about a factor 5) compared to F4 TMS ANT in order to obtain micrographs with comparable PL intensity per pixel. The broad and featureless PL spectrum of F4 TMS ANT is peaked at about $556 \mathrm{~nm}$, with a full width at half maximum of $104 \mathrm{~nm}$ [Fig. 8(a)]; this maximum is red-shifted with respect to the emission maxima observed for TIPS ANT $p$ [see Fig. 8(b)], a feature due to $\pi-\pi$ interactions between anthracene moieties, in accordance with literature [see, e.g.. Teka et al. (2015)]. Spatially resolved PL measurements were performed on single crystals of both samples [Figs. $8(c)$ and $8(d)$ ] with length of about $150 \mu \mathrm{m}$. F4 TMS ANT showed highly uniform emission spectral features as well as intensity along the length of the needle-like system [Fig. 8(c)]. Indeed, the variation $\left(\Delta \lambda_{p}\right)$ of the PL peak wavelength $\left(\lambda_{p}\right)$ along the length of the needle of F4 TMS ANT is $<3 \mathrm{~nm}\left(\Delta \lambda_{p} / \lambda_{p}=\right.$ $0.5 \%)$, and a similar spatial stability is found for the PL full width at half maximum (measured values are in the interval 102-104 nm). The PL spectrum of a single platelet crystal of TIPS ANT $p$ is, instead, much more structured, with peaks at $454 \mathrm{~nm}, 474 \mathrm{~nm}, 500 \mathrm{~nm}, 508 \mathrm{~nm}$ and $538 \mathrm{~nm}$ [Fig. 8(b)]. Some of these PL peaks (the ones at $474 \mathrm{~nm}$ and $508 \mathrm{~nm}$ ) are close to those of the PL spectrum of molecules of TIPS ANT $p$ in solution (see Fig. S3 in the supporting information, where PL peaks at $446 \mathrm{~nm}, 475 \mathrm{~nm}$ and $507 \mathrm{~nm}$ can be identified for the molecule in solution) (del Valle et al., 2002). In addition, polarized PL spectra shown in Fig. S4 in the supporting information highlight a variation of the shape of the emission spectrum of 
TIPS ANT $p$ upon changing the polarization of collected light. This analysis unveils the presence of a peak, in the high energy tail of the PL spectrum, that is polarized in a direction parallel to the short axis of the crystal face, and a dependence of the intensity ratio of the peaks at $500 \mathrm{~nm}$ and $508 \mathrm{~nm}$ on polarization. The structured shape of the spectrum and the polarization dependence are indicative of the presence of different emissive species. Here we point out that the confocal microscopy measurements allow some spectral features of the spectrum below $475 \mathrm{~nm}$ to be unveiled for crystalline samples of TIPS ANTp, which were previously masked by selfabsorption (Camposeo et al., 2019). This is highlighted in Fig. S5a where the emission spectra of TIPS ANTp, measured by vertically shifting the high-numerical objective (NA = 1.42) along the crystal thickness ( $z$ axis in Fig. S5), are compared. This analysis highlights the increasing contribution of selfabsorption as the excitation focal spot is shifted into the crystal, resulting in a decrease of the intensity of the highenergy transitions with respects to the low energy one (Fig. S5b). The larger self-absorption effect in the case of TIPS ANT $p$ is also favoured by the strong overlap between the absorption and PL spectra, more prominent compared to F4 TMS ANT (see Fig. S6). Similarly to F4 TMS ANT, the PL spectrum does not feature significant variations within individual platelet crystal, as obtained by spatially resolved photoluminescence [Fig. $8(d)$ ]. The different shape of the

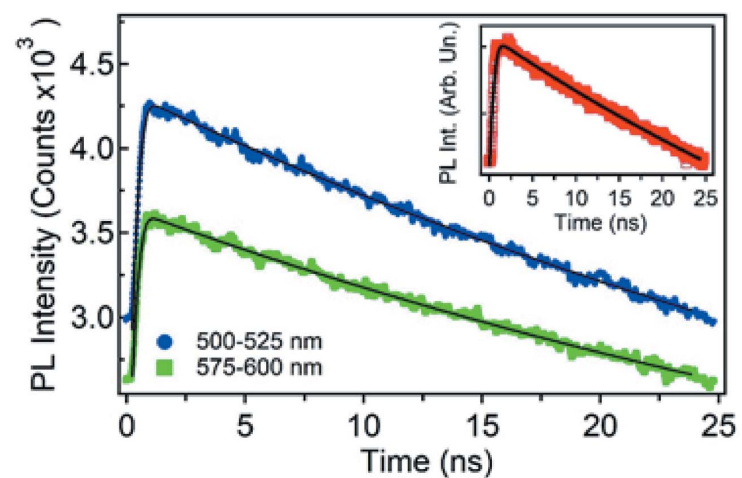

(a)

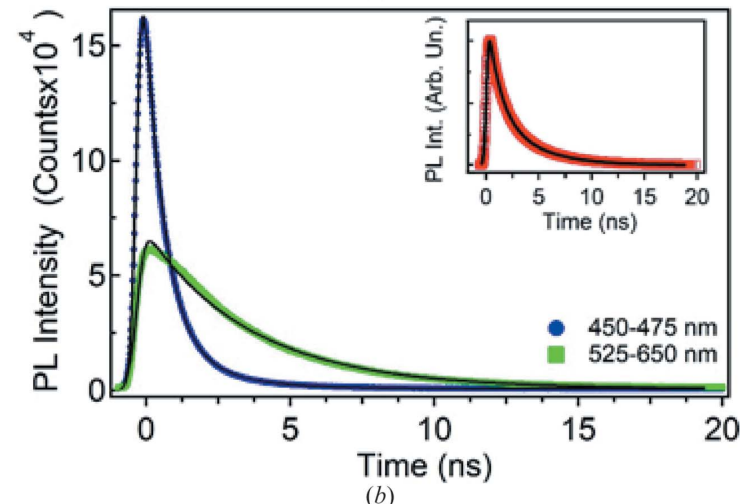

Figure 9

Decay of the PL intensity of F4 TMS ANT (a) and TIPS ANTp (b), in different spectral ranges. The insets of $(a)$ and $(b)$ display the decay of the PL intensity integrated in the whole emission range. The continuous black lines are fits to data by exponential functions convoluted with the instrumental response function. crystalline samples (needle versus platelet) was demonstrated to determine a different light transport behaviour (Camposeo et al., 2019), namely a more efficient self-waveguiding of the emitted light in needles compared to platelets.

The PL lifetime of F4 TMS ANT and TIPS ANT $p$ are shown in Fig. $9(a)$ and $9(b)$, respectively. While a long lifetime in the range $50-80 \mathrm{~ns}$ is estimated for F4 TMS ANT, the PL lifetime of TIPS ANTp is about an order of magnitude shorter, being in the interval 1-4 ns [compare insets of Figs. $9(a)$ and $9(b)$ ]. The longer PL lifetime for F4 TMS ANT could be due to $\pi-\pi$ interactions and the columnar stacking of chromophores. Interestingly, also the spectral dependence of the lifetime is different for the two compounds: the temporal decay of the PL of F4 TMS ANT is constant throughout the spectral range of the emission, whereas for TIPS ANTp we observed an increase of the lifetime by red-shifting the PL wavelengths [Fig. $9(a)$ and $9(b)$ ]. More specifically, the lifetime for the highenergy components of TIPS ANTp (in the interval 450$475 \mathrm{~nm}$ ) is about $0.8 \mathrm{~ns}$, and it increases to about $4 \mathrm{~ns}$ for the low energy ones (525-600 nm). For the sake of comparison, we recall that solutions of TIPS ANT molecules at low concentrations have been reported to show a wavelength-independent lifetime of about $6.6 \mathrm{~ns}$ (Pun et al., 2018). Measurements performed at various $z$-positions of the objective do not evidence significant differences of the PL decay curves (the estimated lifetime is around $2 \mathrm{~ns}$ for all $z$ values), ruling out potential effects related to self-absorption and re-emission (Fig. S7). A similar behaviour of the lifetime was reported also for other acenes, such as anthracene and tetracene (Ahn et al., 2008 and Camposeo et al., 2010), and was attributed to excitonic and defect or trapped states.

Overall, the results of the microscopic PL measurements are consistent with the crystallographic analysis and support the different molecular packing of the investigated compounds in crystalline samples. In fact, F4 TMS ANT crystallizes as elongated needle-like samples, with parallel offset $\pi-\pi$ interaction and columnar stacking of chromophores. These configurations might lead to excimers (Liu et al., 2016) with resulting red-shifted, broad, long-lasting emission. In contrast, the increased separation of the molecules in crystals of TIPS ANT $p$ and the weaker molecular interactions allow the electronic properties of the individual molecules to be partially preserved in the crystalline samples, which show structured PL spectra ascribable to different emitting species.

\section{Conclusions}

The crystal structures of two silylethyne-substituted anthracene compounds [i.e. 1,2,3,4-tetrafluoro-5,8-bis(trimethylsilylethynyl)anthracene and a new polymorph of 9,10bis(triisopropylsilylethynyl)anthracene] were determined by single-crystal synchrotron diffraction to identify main factors influencing the optical properties of these organic semiconductors. The crystallographic study revealed that the two compounds were characterized by different intermolecular interactions, responsible for dissimilar luminescence effects. The crystal morphology also affects the optical properties of 
the two compounds: needle-shape crystals are characterized by $\pi-\pi$ interactions and show broad and long-lasting photoluminescence. This was not found in the case of the second crystal that was platelet-shaped, for which a brighter PL with shorter lifetime was also observed, which could be exploited for the development of efficient light-emitting components and optical sensors.

\section{Funding information}

Funding for this research was provided by: European Research Council under the European Union's Horizon 2020 Research and Innovation Programme (grant No. 682157 to Andrea Camposeo, Dario Pisignano); Italian Minister of University and Research under the PRIN program (grant No. 201795SBA3 to Andrea Camposeo); US National Science Foundation (grant No. 1849213 to John E. Anthony).

\section{References}

Ahn, T.-S., Müller, A. M., Al-Kaysi, R. O., Spano, F. C., Norton, J. E., Beljonne, D., Brédas, J.-L. \& Bardeen, C. J. (2008). J. Chem. Phys. 128, 054505.

Allard, S., Forster, M., Souharce, B., Thiem, H. \& Scherf, U. (2008). Angew. Chem. Int. Ed. 47, 4070-4098.

Anthony, J. E. (2006). Chem. Rev. 106, 5028-5048.

Anthony, J. E. (2008). Angew. Chem. Int. Ed. 47, 452-483.

Anthony, J. E., Eaton, D. L. \& Parkin, S. R. (2002). Org. Lett. 4, 15-18.

Anthony, J. E. \& Parkin, S. R. (2016). CSD Communication.

Bhattacharyya, K. \& Datta, A. (2017). J. Phys. Chem. C, 121, 14121420.

Burla, M. C., Caliandro, R., Carrozzini, B., Cascarano, G. L., Cuocci, C., Giacovazzo, C., Mallamo, M., Mazzone, A. \& Polidori, G. (2015). J. Appl. Cryst. 48, 306-309.

Camposeo, A., Granger, D. B., Parkin, S. R., Altamura, D., Giannini, C., Anthony, J. E. \& Pisignano, D. (2019). Chem. Mater. 31, 17751783.

Camposeo, A., Polo, M., Tavazzi, S., Silvestri, L., Spearman, P., Cingolani, R. \& Pisignano, D. (2010). Phys. Rev. B, 81, 033306.

Chen, Z., Müller, P. \& Swager, T. M. (2006). Org. Lett. 8, 273-276.

Dou, L., You, J., Hong, Z., Xu, Z., Li, G., Street, R. A. \& Yang, Y. (2013). Adv. Mater. 25, 6642-6671.

Farrugia, L. J. (2012). J. Appl. Cryst. 45, 849-854.

Giacovazzo, C. (2014). Phasing in Crystallography: A Modern Perspective. Oxford University Press.

Griffith, O. L., Jones, A. G., Anthony, J. E. \& Lichtenberger, D. L. (2010). J. Phys. Chem. C, 114, 13838-13845.

Gu, X., Yao, J., Zhang, G., Yan, Y., Zhang, C., Peng, Q., Liao, Q., Wu, Y., Xu, Z., Zhao, Y., Fu, H. \& Zhang, D. (2012). Adv. Funct. Mater. 22, 4862-4872.

Hattab, Z., Barbey, C., Monteil, M., Retailleau, P., Aouf, N.-E., Lecouvey, M. \& Dupont, N. (2010). J. Mol. Struct. 973, 144-151.

Ito, K., Suzuki, T., Sakamoto, Y., Kubota, D., Inoue, Y., Sato, F. \& Tokito, S. (2003). Angew. Chem. Int. Ed. 42, 1159-1162.
Janiak, C. (2000). J. Chem. Soc. Dalton Trans. pp. 3885-3896.

Kabsch, W. (2010). Acta Cryst. D66, 125-132.

Kéna-Cohen, S. \& Forrest, S. R. (2010). Nat. Photonics 4, 371375.

Kovalski, E., Korb, M. \& Hildebrandt, A. (2018). Eur. J. Inorg. Chem. 5, 617-675.

Landis, C. A., Parkin, S. R. \& Anthony, J. E. (2005). Jpn. J. Appl. Phys. 44, 3921-3922.

Liu, H., Yao, L., Li, B., Chen, X., Gao, Y., Zhang, S., Li, W., Lu, P., Yang, B. \& Ma, Y. (2016). Chem. Commun. 52, 7356-7359.

Liu, Z., Xiao, J., Fu, Q., Feng, H., Zhang, X., Ren, T., Wang, S., Ma, D., Wang, X. \& Chen, H. (2013). Appl. Mater. Interfaces, 5, 1113611141.

Macrae, C. F., Bruno, I. J., Chisholm, J. A., Edgington, P. R., McCabe, P., Pidcock, E., Rodriguez-Monge, L., Taylor, R., van de Streek, J. \& Wood, P. A. (2008). J. Appl. Cryst. 41, 466-470.

Martinez, C. R. \& Iverson, B. L. (2012). Chem. Sci. 3, 21912201.

Mei, J., Diao, Y., Appleton, A. L., Fang, L. \& Bao, Z. (2013). J. Am. Chem. Soc. 135, 6724-6746.

Meyer, E. A., Castellano, R. K. \& Diederich, F. (2003). Angew. Chem. Int. Ed. 42, 1210-1250.

Nishio, M. (2004). CrystEngComm, 6, 130-158.

Nishio, M., Umezawa, Y., Suezawa, H. \& Tsuboyama, S. (2012). The $C-H \cdots \pi$ Hydrogen Bond: Implication in Crystal Engineering. In The Importance of Pi-Interactions. In Crystal Engineering: Frontiers in Crystal Engineering, 1st ed., edited by E. R. T. Tiekink and J. Zukerman-Schpector. John Wiley \& Sons.

Ostroverkhova, O. (2016). Chem. Rev. 116, 13279-13412.

Palilis, L. C., Lane, P. A., Kushto, G. P., Purushothaman, B., Anthony, J. E. \& Kafafi, Z. H. (2008). Org. Electron. 9, 747-752.

Pun, J. K. H., Gallaher, J. K., Frazer, L., Prasad, S. K. K., Dover, C. B., MacQueen, R. W. \& Schmidt, T. W. (2018). J. Photon. Energ. 8, 022006.

Sheldrick, G. M. (2015). Acta Cryst. A71, 3-8.

Silva Filho, D. da, Kim, E.-G. \& Brédas, J.-L. (2005). Adv. Mater. 17, 1072-1076.

Teka, S., Hriz, K., Jaballah, N., Kreher, D., Mathevet, F., Jarroux, N. \& Majdoub, M. (2015). Mater. Sci. Semicond. Process. 34, 189197.

Turner, M. J., McKinnon, J. J., Wolff, S. K., Grimwood, D. J., Spackman, P. R., Jayatilaka, D. \& Spackman, M. A. (2017). CrystalExplorer17. University of Western Australia. http://hirshfeldsurface.net.

Valle, J. C. del, Turek, A. M., Tarkalanov, N. D. \& Saltiel, J. (2002). J. Phys. Chem. A, 106, 5101-5104.

Waltersperger, S., Olieric, V., Pradervand, C., Glettig, W., Salathe, M., Fuchs, M. R., Curtin, A., Wang, X., Ebner, S., Panepucci, E., Weinert, T., Schulze-Briese, C. \& Wang, M. (2015). J. Synchrotron Rad. 22, 895-900.

Wang, S., Kappl, M., Liebewirth, I., Müller, M., Kirchhoff, K., Pisula, W. \& Müllen, K. (2012). Adv. Mater. 24, 417-420.

Westrip, S. P. (2010). J. Appl. Cryst. 43, 920-925.

Yao, Z.-F., Wang, J.-Y. \& Pei, J. (2018). Cryst. Growth Des. 18, 7-15. Yersin, H. (2008). Editor. Highly Efficient OLEDs with Phosphorescent Materials. WILEY-VCH Verlag GmbH \& Co. KGaA, Weinheim. 